\author{
Alba Alfaro López ${ }^{1}$ \\ Evelin Balaguer López² \\ Alberto Pérez Paredes, ${ }^{3, *}$ \\ Juan Gómez Salgado ${ }^{4}$ \\ Mari Carmen Rodríguez Dolz ${ }^{5}$ \\ Pablo García Molina ${ }^{6}$
}

1. Enfermera asistencial. Universitat de València. Valencia. España.

2. Enfermera, Servicio de Pediatría, Hospital Clínico Universitario de Valencia Especialista en Enfermería Pediátrica. Profesora del Departamento Enfermería, Universitat de València (UV), Valencia, España. Miembro del Grupo de Investigación Cuidados Enfermeros (INCLIVA). Editora upppediatria.org.

3. Enfermero. Máster en Enfermería Oncológica. Colaborador investigador de la Unidad Enfermera de Úlceras y Heridas complejas. Hospital Clínico Universitario de Valencia. Valencia. España.

4. Doctor por la Universidad de Huelva. Profesor Ayudante Doctor, Facultad de Enfermería, Universidad de Huelva, Huelva, España. Universidad Espíritu Santo, Guayaquil, Ecuador.

5. Enfermera. Servicio de Pediatría, Hospital Clínico Universitario de Valencia, Valencia, España. Especialista en Enfermería Pediátrica. Profesora Departamento Enfermería, Universitat de València. Miembro del Grupo de Investigación Cuidados Enfermeros (INCLIVA).

6. Doctor por la Universidad de Alicante. Especialista Enfermería Pediátrica. Profesor del Departamento Enfermería, Universitat València, Valencia, España. Junta Directiva SEEN. Comité Consultivo GNEAUPP. Editor upppediatria.org. Miembro del Grupo de Investigación Cuidados Enfermeros (INCLIVA). Colaborador investigador de la Unidad Enfermera de Úlceras y Heridas complejas. Hospital Clínico Universitario de Valencia.

\section{Efectividad de las medidas y estrategias de prevención de úlceras por presión en neonatos}

\section{Effectiveness of measures and strategies for the prevention of pressure ulcers in neonates}

*Autor para correspondencia.

Correo electrónico: alpe1591@gmail.com (Alberto Pérez Paredes)

Recibido el 15 de enero de 2019; aceptado el 11 de noviembre de 2019

\section{RESUMEN}

Objetivos: Analizar la efectividad, en términos de disminución de la incidencia de úlceras por presión (UPP), de las medidas y estrategias preventivas de UPP en neonatos hospitalizados. Método: La recuperación de estudios se realizó a través de tres bases de datos (Medline a través de PubMed, Scopus y Science Direct) y a partir de un experto. Solamente se incluyeron en esta revisión los estudios publicados del 2007 al 2017 en español o inglés, que de forma directa o indirecta valoraran la efectividad de una medida o estrategia preventiva de UPP en neonatos hospitalizados y que en cuanto al diseño fueran o estudios clínicos controlados o cuasiexperimentales. Resultados: De 100 estudios recuperados se realizó el análisis de cinco; los demás fueron excluidos por no cumplir los criterios de elegibilidad. La mayoría de los estudios analizan la efectividad de una medida preventiva de UPP secundaria al tratamiento con ventilación mecánica no invasiva en neonatos prematuros. Todos ellos tienen limitaciones en la metodología que emplean, lo que hace que la evidencia de sus recomendaciones sea baja-moderada. Conclusiones: Es necesaria la realización de estudios controlados aleatorios de mayor potencia para poder recomendar alguna medida o estrategia preventiva.

\section{ABSTRACT}

Objetives: The main objective of this review was to examine the effectiveness, in terms of reducing the incidence of pressure ulcers (PU), of measures and preventive strategies of PU in hospitalized infants. Method: The recovery of studies was carried out through three databases (Medline through Pubmed, Scopus and Science Direct), and from an expert. Only studies published from 2007-2017 in Spanish or English were included in this review, which directly or indirectly appreciate the effectiveness of a measure or preventive strategy of PU in hospitalized neonates and that in terms of design were controlled or quasi-experimental trials. Results: From 100 retrieved studies was conducted analysis of 5 studies, others were excluded for failing to meet the eligibility criteria. Most studies analyze the effectiveness of a preventive measure of secondary PU to treatment with Noninvasive mechanical ventilation in preterm infants. All of the studies have limitations in the methodology used, which makes the evidence of its recommendations to be low-moderate. Conclusions: Is necessary the realization of randomized controlled studies of higher power to be able to recommend any measure or preventive strategy.

PALABRAS CLAVE: Neonatos, úlcera por presión, prevención.

KEYWORDS: Neonates, pressure ulcer, prevention. 


\section{У INTRODUCCIÓN}

En la actualidad es frecuente que las prácticas de cuidado de la piel de los recién nacidos (RN) estén basadas en la experiencia profesional, la tradición o la adaptación de las pautas de cuidado de la piel en adultos. No obstante, las investigaciones actuales demuestran que el mal cuidado de la piel de los neonatos puede afectar a la salud de estos ${ }^{1-3}$.

La piel es un órgano complejo y dinámico, cuya principal función es servir de barrera entre la persona y el ambiente físico, químico y/o biológico. Así pues, la piel tiene un papel vital en la protección mecánica, en la termorregulación y en la protección de patógenos y tóxicos 4 . La maduración cutánea, tanto a nivel funcional como estructural, es un proceso dinámico. En los $\mathrm{RN}$ a término este proceso comienza inmediatamente después del parto, con la adaptación de la piel del neonato al ambiente seco en comparación con el medio intrauterino, y finaliza en el primer año de vida. Sin embargo, en los $\mathrm{RN}$ prematuros el proceso no se inicia, equiparándose a los $\mathrm{RN}$ a término, hasta pasadas 2-3 semanas posparto, o más tardío si el neonato nace entre las 23-25 semanas de gestación ${ }^{1,5,6}$.

Los factores de riesgo de úlceras por presión (UPP) en la población neonatal hospitalizada son similares a los de la población adulta y tercera edad. Sin embargo, debido a las condiciones anatómicas y fisiológicas de los neonatos, existen factores de riesgo que se acentúan en esta población ${ }^{3,7,8}$.

En la actualidad, no existen apenas investigaciones publicadas sobre medidas preventivas de UPP en neonatos. Por lo tanto, las medidas y estrategias de prevención actuales no disponen de una elevada evidencia científica, ya que la mayoría están basadas en opinión de expertos o adaptadas de investigaciones en adultos ${ }^{9-11}$. Este hecho hace que las recomendaciones actuales sean muy diversas y no basadas en pruebas fehacientes $^{7,12}$. Debido a esto, nos planteamos realizar un estudio de revisión sistemática, para analizar la efectividad, en términos de disminución de la incidencia de UPP, de las medidas y estrategias preventivas de UPP en neonatos hospitalizados.

\section{Y OBJETIVO}

Analizar la efectividad, en términos de disminución de la incidencia de UPP, de las medidas y estrategias preventivas de UPP en neonatos hospitalizados.

\section{У MÉTODOS}

\section{Diseño}

Para la consecución de los objetivos propuestos, se realizó una revisión sistemática siguiendo las recomendaciones de la Cochrane Collaboration, utilizando el método PRISMA ${ }^{13}$ para la realización de la revisión.

\section{Criterios de inclusión}

Solamente se incluyeron estudios clínicos controlados y cuasiexperimentales. Se incluyeron aquellos estudios cuya población fuera neonata en ámbito hospitalario, que se refiriesen directa o indirectamente a algún tipo de intervención preventiva de UPP y que hubieran sido publicados en español o inglés en un intervalo de tiempo de 10 ańos, desde 2007 hasta 2017.

La elaboración de la pregunta de revisión se definió según la metodología PICOS (Population, Intervention, Comparisons, Outcomes y Study Design). Este método permitió identificar de forma sencilla los componentes de la pregunta a estudio (tabla 1).
Tabla 1. Componentes de la pregunta de revisión según el método PICOS

\begin{tabular}{ll}
\hline P & $\begin{array}{l}\text { Neonatos hospitalizados } \\
\text { Medidas o estrategias de prevención de úlceras por presión } \\
\text { (UPP) }\end{array}$ \\
\hline Comparación entre los neonatos que reciben medidas \\
de prevención de UPP y aquellos que no reciben medidas \\
preventivas de UPP
\end{tabular}

Fuente: Elaboración propia

Tabla 2. Estrategia de búsqueda en Medline

("Pressure Ulcer"[Mesh] OR "decubit ulcer" OR "pressure sore" OR bedsore OR "injury skin" OR "skin breakdown")

(prevention OR mattresses OR "support surface" OR "pressure management" OR dressing OR "prevention program"

\#2 OR repositioning $O R$ nutrition OR diet OR "skin hygiene" OR "hyperoxygenated oils" OR "humidity management" OR "hydration of the skin")

\#3

(neonate OR newborn OR "Infant, Newborn" [Mesh] OR "premature newborn")

\#1 AND \#2 AND \#3

[/“Pressure Ulcer"[Mesh] OR "decubit ulcer" OR "pressure sore" OR bedsore OR "injury skin" OR "skin breakdown") AND (prevention OR mattresses OR "support surface" OR "pressure

\#4 management" OR dressing OR "prevention program" OR repositioning $\mathrm{OR}$ nutrition $\mathrm{OR}$ diet $\mathrm{OR}$ "skin hygiene" $O R$ "hyperoxygenated oils" OR "humidity management" OR "hydration of the skin") AND (neonate OR newborn OR "Infant, Newborn"[Mesh] OR "premature newborn")]

Fuente: Elaboración propia

\section{Fuentes de información y estrategia de búsqueda}

Se realizaron búsquedas en tres bases de datos anglosajonas: Medline a través de PubMed, Scopus y Science Direct. La estrategia de búsqueda se realizó basándose en los tres conceptos claves de la revisión: UPP, medidas/intervenciones preventivas de UPP y neonatos, sin realizarse una búsqueda inversa en las listas de bibliografía. En la tabla 2 se puede observar la estrategia de búsqueda utilizada para la base de datos Medline a través de la web de PubMed.

\section{Criterio de selección de los estudios}

Dos revisores procedieron a la lectura del título y el resumen de la totalidad de referencias recuperadas para seleccionar los documentos que pasarían a la siguiente fase. La valoración se hacía indicando sí, no, o dudoso.

Posteriormente, los artículos catalogados con sí o dudoso fueron leídos por otro revisor, que junto a los otros dos revisores selecciona- 
ron la muestra de artículos incluidos. El resto de los miembros del equipo de investigación estaban encargados de la organización administrativa y gestora del estudio. Dos de los investigadores, además, actuaron como profesionales relevantes en el ámbito de la pediatría.

\section{$\checkmark$ RESULTADOS}

Tras la búsqueda en las diferentes bases de datos se recuperaron 98 estudios. De los estudios recuperados, 42 estudios se obtuvieron de Medline a través de PubMed, 44 estudios de Scopus y 12 a través de la base de datos Science Direct (fig. 1).

De los cinco estudios incluidos en la revisión, tres fueron estudios clínicos controlados ${ }^{14-16}$ y dos, cuasiexperimentales ${ }^{17,18}$.

El objetivo de dos de los estudios clínicos controlados ${ }^{14,15}$ fue comprobar la efectividad de medidas preventivas de UPP secundarias a la utilización de tratamiento con ventilación mecánica no invasiva (VMNI) de un apósito de protección. El otro estudio clínico controlado $^{16}$ tuvo como objetivo principal identificar las diferencias en cuanto a frecuencia y gravedad de las UPP secundarias al tratamiento

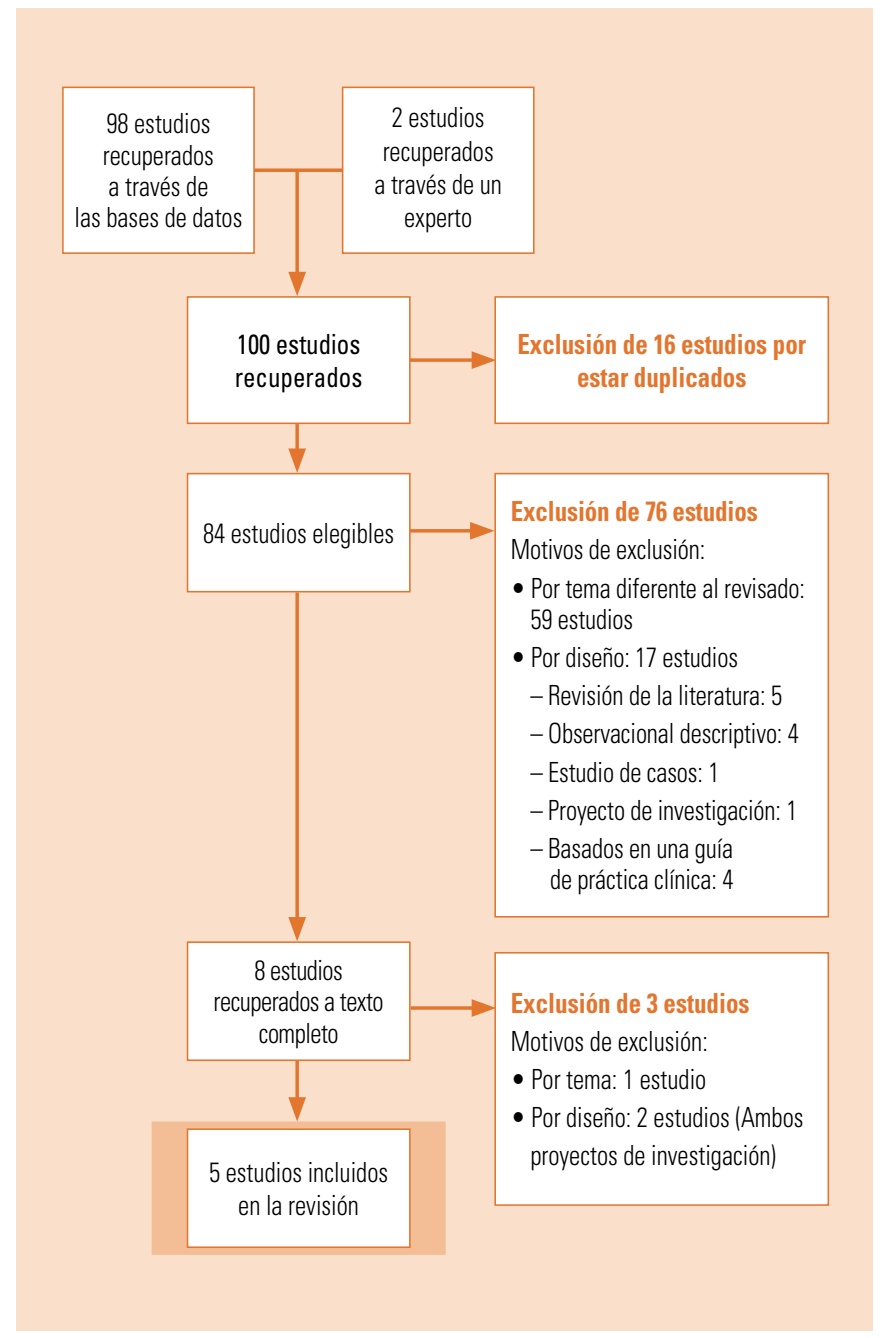

Figura 1. Diagrama de flujo. Fuente: elaboración propia. con VMNI de diferentes dispositivos utilizados en el tratamiento con VMNI.

En cuanto a los estudios cuasiexperimentales, el objetivo de uno de ellos ${ }^{17}$ fue comparar cinco superficies de apoyo neonatales utilizadas frecuentemente en las unidades de cuidados intensivos neonatales (UCIN) para determinar sí existen diferencias en cuanto a la presión que estos ejercen en la zona occipital. El objetivo del estudio restante ${ }^{18}$ fue evaluar el efecto de la aplicación de un paquete de medidas preventivas de UPP para reducir la incidencia de UPP en neonatos.

La población analizada incluyendo los cinco estudios ${ }^{14-18}$ suma un total de 732 neonatos. La edad gestacional media de la muestra de todos los estudios fue inferior a 37 semanas $^{14-17}$, excepto en un estudio ${ }^{18}$, en el cual los neonatos tuvieron una gestación media superior a 37 semanas. En cuanto al peso, tres estudios ${ }^{14-16}$ hicieron referencia al peso al nacer, un estudio $^{17}$ al peso del neonato en el momento de la inscripción al estudio y otro $^{18}$ no ofreció ningún dato relacionado con el peso. La media de peso al nacer en los tres estudios ${ }^{14-16}$ que reflejaron este dato fue inferior a 1.800 g. En el estudio en el que se consideró el peso que tenía un neonato en el momento de realizar el estudio, la media fue mayor de $2.500 \mathrm{~g}^{17}$.

En la tabla 3 se pueden ver los principales resultados de los estudios analizados.

Tabla 3. Resultados principales de los estudios analizados

\begin{tabular}{|c|c|c|}
\hline Estudio & Resultado & $\begin{array}{c}\text { Datos } \\
\text { estadísticos }\end{array}$ \\
\hline $\begin{array}{l}\text { Günlemez } \\
\text { et al., 200837 }\end{array}$ & $\begin{array}{l}\text { Incidencia de UPP: } \\
\text { GC: } 4,3 \%(n=4) \\
\text { GE: } 14,9 \%(n=13) \\
\text { Incidencia de necrosis: } \\
\text { GC: } 6,8 \% \\
\text { GE: } 1,08 \%\end{array}$ & $\begin{array}{l}\text { OR: } 3,43 ; \text { IC } 95 \%: \\
\text { 1,1-10,1; } p<0,05 \\
\text { OR: } 6,34 ; \text { IC } 95 \% \text { : } \\
\text { 0,78-51,6; } \\
\quad p<0,05\end{array}$ \\
\hline $\begin{array}{l}\text { Xie et al., } \\
2014^{15}\end{array}$ & $\begin{array}{l}\text { Incidencia de UPP: } \\
\text { GC: } 21,8 \%(n=7) \\
\text { GE: } 6 \%(n=2) \\
\text { Gravedad UPP: } \\
\text { GC: } 6 \text { RN UPP leve y } 1 \text { RN UPP grave } \\
\text { GE: } 2 \text { RN UPP leve-moderada }\end{array}$ & $p=0,01$ \\
\hline $\begin{array}{l}\text { Newnam } \\
\text { et al., 2015 }\end{array}$ & $\begin{array}{l}\text { Incidencia de UPP total: 24,2\% } \\
\text { Incidencia UPP menor en el grupo } \\
\text { de rotación de cánulas } \\
\text { nasales-mascarilla }\end{array}$ & $p<0,05$ \\
\hline $\begin{array}{l}\text { Turnage- } \\
\text { Carrier } \\
\text { et al., 2008 }\end{array}$ & $\begin{array}{l}\text { Presión media (mmHg): } \\
\text { Colchón de cuna estándar: 86,9 } \\
\text { Colchón de gel: } 49,8 \\
\text { Almohada de gel tipo Gel-E-donut } \\
\text { tamaño infantil: } 37,5 \\
\text { Almohada de agua (288 ml): 50,1 } \\
\text { Colchón de superposición-Delta de } \\
\text { espuma: } 31\end{array}$ & $(\mathrm{IC} 95 \% ; p<0,05)$ \\
\hline $\begin{array}{l}\text { Schindler } \\
\text { et al., 2013 }\end{array}$ & $\begin{array}{l}\text { Incidencia de UPP: } \\
\text { GC: } 18,8 \%(n=28) \\
\text { GE: } 6,8 \%(n=17)\end{array}$ & $p<0,001$ \\
\hline
\end{tabular}

GC: grupo control, GE: grupo experimental, IC: intervalo de confianza; OR: odds ratio; RN: recién nacido; UPP: úlceras por presión.

Fuente: Elaboración propia 


\section{Medidas preventivas de UPP en neonatos hospitalizados}

De los cinco estudios analizados ${ }^{14-18}$, no se encontró ninguno cuyo objetivo principal fuera valorar la efectividad, en términos de disminución de la incidencia de UPP en neonatos hospitalizados, de una medida preventiva de UPP producidas por presión convencional. No obstante, sí que se analizó un estudio, el de Turnage-Carrier et al. ${ }^{17}$, cuyo objetivo fue determinar la presión de contacto entre el occipucio de neonatos sanos y cinco superficies de apoyo utilizadas con frecuencia en las UCIN. El objetivo de este estudio ${ }^{17}$ de forma indirecta fue determinar qué tipo de superficie de apoyo era más adecuada para los neonatos con largas estancias en las UCIN.

La intervención realizada en dos de los estudios, Günlemez et al. ${ }^{14} \mathrm{y}$ Xie et al. ${ }^{15}$, fue la colocación de un apósito de protección previo al inicio de VMNI. En uno de los estudios ${ }^{14}$ se empleó un apósito de gel de silicona, mientras que en el otro estudio ${ }^{15}$ se utilizó un apósito hidrocoloide. Ambos estudios ${ }^{14,15}$ disminuyeron la incidencia de UPP secundaria al tratamiento con VMNI mediante su intervención a estudio. En ambos casos, la incidencia fue significativamente menor en el grupo experimental respecto al grupo control $(p<0,05)$. También valoraron el intervalo de tiempo entre el inicio de VMNI y la aparición de lesión. Sin embargo, solamente en uno de los estudios ${ }^{14}$ el intervalo de tiempo fue estadísticamente mayor en el grupo experimental versus el grupo control $(p<0,05)$.

\section{Estrategias preventivas de UPP en neonatos hospitalizados}

Solamente uno de los estudios analizados, el de Schindler et al. ${ }^{18}$, investigó la efectividad de la aplicación de un conjunto de medidas preventivas, es decir de una estrategia de prevención. Los resultados de este estudio determinaron una incidencia menor en el grupo que recibió las intervenciones preventivas. Las intervenciones realizadas en este estudio se fundamentaron en cinco aspectos: superficie de apoyo adecuada para la disminución de presión de contacto, reposicionamiento frecuente, control y gestión de la incontinencia, nutrición adecuada y educación a los profesionales.

\section{Riesgo de sesgo de los estudios}

Ninguno de los estudios controló todos los posibles factores de confusión. En la tabla 4 se puede observar la justificación de la evaluación del riesgo de sesgo de los estudios analizados.

\section{У DISCUSIÓN}

Respecto a las medidas preventivas de UPP en neonatos, hemos observado que la mayoría de los estudios son observacionales descripti$\operatorname{vos}^{19-21}$. Entre los hallazgos principales en nuestra revisión ninguna de las medidas preventivas analizadas puede recomendarse por encima de otra basada en opinión de expertos. No existen pruebas suficientes para determinar la efectividad de las medidas y estrategias preventivas.

Existen una mayor cantidad de estudios cuyo objetivo es valorar la efectividad de una medida preventiva de UPP secundaria al uso de dispositivos clínicos, en concreto secundarias a los dispositivos empleados en tratamiento de VMNI ${ }^{11-13}$ en comparación con aque$\operatorname{llos}^{14,15}$ que analizan medidas preventivas de UPP (superficies especiales para el manejo de la presión, cambios posturales) producidas por presión convencional, entendida esta como la presión de contacto entre el paciente y una superficie de apoyo, y excluyendo de esta definición la presión ejercida por un dispositivo clínico.

Las principales limitaciones observadas en los estudios analizados se centran en la obtención de la muestra, pues en todos los estudios ${ }^{11-15}$ el muestreo empleado es por conveniencia, lo que podría suponer una muestra no representativa. Además, este hecho se acentúa en $\operatorname{dos}^{11,12}$ de los estudios, pues tampoco realizaron cálculo muestral. Otra de las limitaciones importantes

Tabla 4. Evaluación del riesgo de sesgo

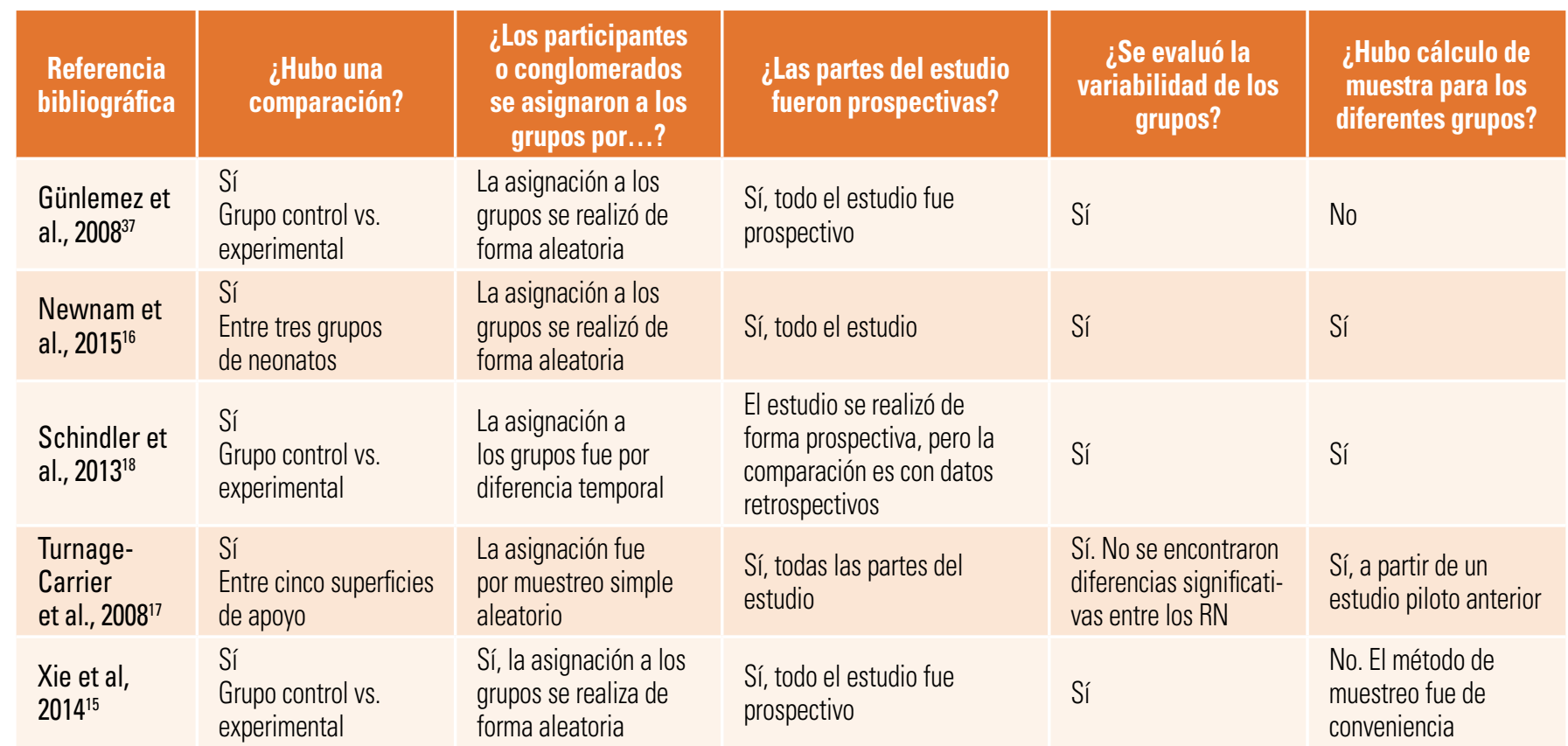

Fuente: elaboración propia a partir del modelo de la Fundación Cochrane. 
observada en algunos de los estudios ${ }^{12,13,15}$ es la no comparabilidad de los grupos a estudio, pues se encuentran diferencias significativas que podrían sesgar los resultados obtenidos en el estudio. Estas limitaciones implican una disminución de la calidad de los estudios y por tanto de la evidencia científica que ofrecen sus resultados y recomendaciones.

Como limitaciones de la revisión encontramos la lingüística. Tan solo se aceptaron artículos en español e inglés. A su vez, se decidió no incluir la base de datos Scielo, lo que constituye un sesgo a la hora de recuperar artículos en portugués.

La metodología de la Cochrane Collaboration recomienda que al menos dos revisores intervengan en las fases de búsqueda bibliográfica y en la selección de estudios, para poder realizar una revisión sistemática de calidad. En nuestro estudio, la búsqueda bibliográfica fue realizada por un miembro del equipo.

\section{$\searrow$ CONCLUSIONES}

Ninguna de las medidas preventivas analizadas en esta revisión puede recomendarse por encima de otra, ya que no hay evidencia suficiente para determinar la efectividad de las medidas y estrategias preventivas.

Para futuras investigaciones, es necesaria la realización de estudios clínicos controlados y aleatorizados de calidad y con cálculo de muestra que valoren la efectividad de las medidas preventivas. También es recomendable que los investigadores analicen las intervenciones preventivas en grupos comparables entre sí y que disgreguen los resultados según la edad gestacional

\section{Conflicto de intereses}

Los autores declaran no tener ningún conflicto de intereses.

\section{У BIBLIOGRAFÍA}

1. Oranges T, Dini V, Romanelli M. Skin Physiology of the Neonate and Infant: Clinical Implications. Adv Wound Care. 2015;4(10):587-95.

2. Ness MJ, Davis DMR, Carey WA. Neonatal skin care: a concise review. Int J Dermatol. 2013:52(1):14-22.

3. Garcia-Molina P, Balaguer-Lopez E, Garcia-Fernandez FP, Ferrera-Fernandez M de LA, Blasco JM, Verdu J. Pressure ulcers' incidence, preventive measures, and risk factors in neonatal intensive care and intermediate care units. Int Wound $\mathrm{J}$. 2018;15(4):571-9.

4. Afsar FS. Physiological skin conditions of preterm and term neonates. Clin Exp Dermatol. 2010;35(4):346-50.

5. Scheans P. Neonatal Pressure Ulcer Prevention. Neonatal Netw. 2015;34(2):126-32.

6. García-Molina P, Alfaro-López A, García-Rodríguez SM, Brotons-Payá C, Rodríguez-Dolz MC, Balaguer-López E. Neonatal pressure ulcers: prevention and treatment. Res Reports Neonatol. 2017;7:29-39.

7. Quesada-Ramos C, García-Molina P. Úlceras por presión en pediatría. En: García-Fernández FP, Soldevilla-Ágreda JJ, Torra i Bou J-E, eds. Atención Integral de las Heridas Crónicas. Logroño: Grupo Nacional para el Estudio y Asesoramiento en Úlceras por Presión y Heridas Crónicas: 2016. p. 760.

8. Alepuz Vidal L, Benítez Martínez JC, Casaña Granell J, Clement Imbernón J, Fornes Pujalte B, García Molina P. Guía de práctica clínica para el cuidado de personas con úlceras por presión o ries- go de padecerlas. Conselleria de Sanitat, Generalitat Valenciana, 2012.

9. Baharestani MM, Ratliff CR. Pressure ulcers in neonates and children: an NPUAP white paper. Adv Skin Wound Care. 2007:20(4):208, 210, 212, 214, 216, 218-20.

10. National Pressure Ulcer Advisory Panel, European Pressure UIcer Advisory Panel PPPIA. Prevention and Treatment of Pressure Ulcers: Clinical Practice Guideline [Internet]. En: Haesler E, ed. Osborne Park, Western Australia: Cambridge Media; 2014. Disponible en: https://www.npuap.org/wp-content/uploads/2014/08/ Updated-10-16-14-Quick-Reference-Guide-DIGITAL-NPUAPEPUAP-PPPIA-160ct2014.pdf

11. García-Molina P, Balaguer-López E. Cuidados de la piel. En: Sellán Soto MC, Vázquez Sellán A, eds. Cuidados Neonatales en enfermería. Madrid: Elsevier; 2016. p. 312.

12. Stansby G, Avital L, Jones K, Marsden G. Prevention and management of pressure ulcers in primary and secondary care : summary of NICE. BMJ. 2014;2592(Aprill):1-5.

13. Higgins JPT, Green SE. Manual Cochrane de Revisiones Sistemáticas de Intervenciones, versión 5.1. 0. Man Cochrane Revis Sist Interv versión 510 [Internet]. 2012;(March):1-639. Disponible en: http://www.cochrane.es/?q=es/node/269

14. Gunlemez A, Isken T, Gokalp AS, Turker G, Arisoy EA. Effect of silicon gel sheeting in nasal injury associated with nasal CPAP in preterm infants. Indian Pediatr. 2010;47(3):265-7.

15. Xie L-H. Hydrocolloid dressing in preventing nasal trauma se- condary to nasal continuous positive airway pressure in preterm infants. World J Emerg Med. 2014:5(3):218-22.

16. Newnam KM, McGrath JM, Salyer J, Estes T, Jallo N, Bass WT. A comparative effectiveness study of continuous positive airway pressure-related skin breakdown when using different nasal interfaces in the extremely low birth weigh neonate. Appl Nurs Res [Internet]. 2015;28(1):36-41. Disponible en: http://search.ebscohost.com/login.aspx?direc$t=t r u e \& d b=c i n 20 \& A N=103753179 \&$ lang $=$ es\&site=ehost $-\mathrm{li}-$ ve\&scope $=$ site

17. Turnage-Carrier C, McLane KM, Gregurich MA. Interface pressure comparison of healthy premature infants with various neonatal bed surfaces. Adv Neonatal Care. 2008:8(3):176-84

18. Schindler CA, Mikhailov TA, Cashin SE, Malin S, Christensen M, Winters JM. Under pressure: Preventing pressure ulcers in critically ill infants. J Spec Pediatr Nurs. 2013;18(4):329-41.

19. Fujii K, Sugama J, Okuwa M, Sanada H, Mizokami Y. Incidence and risk factors of pressure ulcers in seven neonatal intensive care units in Japan: a multisite prospective cohort study. Int Wound J. 2010;7(5):323-8

20. August DL, Edmonds L, Brown DK, Murphy M, Kandasamy Y. Pressure injuries to the skin in a neonatal unit: Fact or fiction. J Neonatal Nurs [Internet]. 2014;20(3):129-37. Disponible en: http://www. sciencedirect.com/science/article/pii/S1355184113001294

21. Visscher M, Taylor T. Pressure ulcers in the hospitalized neonate: Rates and risk factors. Sci Rep. 2014;4. 